\title{
IMPLEMENTATION OF LINE TRACING ALGORITHM FOR PATH TRACKING ROBOT
}

\author{
Md Zabiuddin ${ }^{1}$, Pushpalatha $\mathbf{R}^{2}$ \\ ${ }^{1}$ Student, Computer Science and Engineering, VTU PG centre, Mysore, Karnataka, India \\ ${ }^{2}$ Assistant professor, Computer Science and Engineering, VTU PG centre, Mysore, Karnataka, India
}

\begin{abstract}
In the existing line follower robot, the robot operates as the name specifies. It is programmed to run on a black path on a white area and detects the turns and deviations and changes its movement appropriately. Arrays of IR sensors are used for detecting the path. Proposed project designed to work similar to line tracking robot. With slight modification, that reduces the complexity. By eliminating path, background and sensors we have reduced the complexity present in the existing system. RF technology is used in this project that helps in transferring the path drawn on the computer screen for the movement of Robot. The window to draw the line on computer screen is developed using C\# language. And for tracing the path, we have used line algorithm which is aim of this project are used. And the robot is developed by using embedded application. H-bridge circuit is used to control the speed of the robot. Motor is used for the turning movement of Robot. From the computer, the path is transmitted and sensed by microcontroller. For turning of robot, differential steering is used. For each front and back wheel motors are used to rotate along the path. For controlling the two DC motors to navigate through its path, microcontroller is used. The mechanical constructional design and circuitry interfacing with microcontroller of robot has to be presented in this project along with computer side programming.
\end{abstract}

Keywords-IR-Infrared; RF-Radio frequency; DC-Direct Current; D2MASC-design and develop a multipurpose autonomous smart car; CBLFRURF-Command based line following robot using RF technology.

****.

\section{INTRODUCTION}

The Flexible Manufacturing System (FMS) is an advanced production system in which many different standards may be used and many product types can be produced in the same line, and is controlled by computers and equipped with a transportation system that will deliver any work piece to any machine in any sequence [1]-[4]. The FMSs are equipped with several CNC machines and Automatic Guided Vehicle $(\mathrm{AGV})$. An AGV based material handling system is designed and implemented to gain production the flexibility and efficiency. The major difference between an FMS and a conventional job shop is that the human tasks are automated in the FMS. On the other hand, the external guidance signals are understood by $\mathrm{AGV}$ in order to deliver a unit load from origin to destination. Hence AGV functions as an unnamed computerized system. [5].

The AGV's, following markers or wires in the floor are used to transport goods around warehouses, container ports, or hospitals. There are different types of AGV like Intelligent AGV's (i-AGVs). D2MASC is similar to AGV but here it treats the deviation from the track as error and the chosen algorithm serves to minimize this error this leads to reduce in traverse time, distance, power consumption. The proportional integral and derivative control mechanism is used for having better efficiency [9].

DIDLFR is similar to LFR but here differential steering is used for turning robot. In this system, dedicated motor has attached to each back wheel while the front wheel is free to rotate [10]. CBLFRURF is also preprogrammed microcontroller on board as in the traditional LFR but here we are interfacing the computer to give the commands to robot through RF [8].

The line follower is a self-operating robot that detects and follows a line that is drawn on the floor. The path consists of a black path on a white area. The control system used must be capable of following a line and maneuver the robot for staying on course, while the physical path is given to the robot. very tight curves should be followed by the robot. While developing a mobile robot, it should be noticed carefully that the robot should navigate freely and dynamically in the workspace. By new emerging problem or task comes to the need or invention of new techniques, in order to sense or detect the line which is to be followed various ways of path providing mechanism to the robot. When a path from the computer is given to the microcontroller which is fixed on PCB (printed circuit board) and this path is transmitted to H-bridge through the RFmodule then the wheels are controlled and moved.

The objective of this project is to present the model for path tracking for a mobile robot, which would be capable of following a given any path complex or simple. Usually a line single path drawn on the floor is followed by the line follower robot but in this project path is drawn on the computer. This is to avoid the wrong moves which will be traced by the sensors on board due to dark light or cross corners. 


\section{ARCHITECTURE}
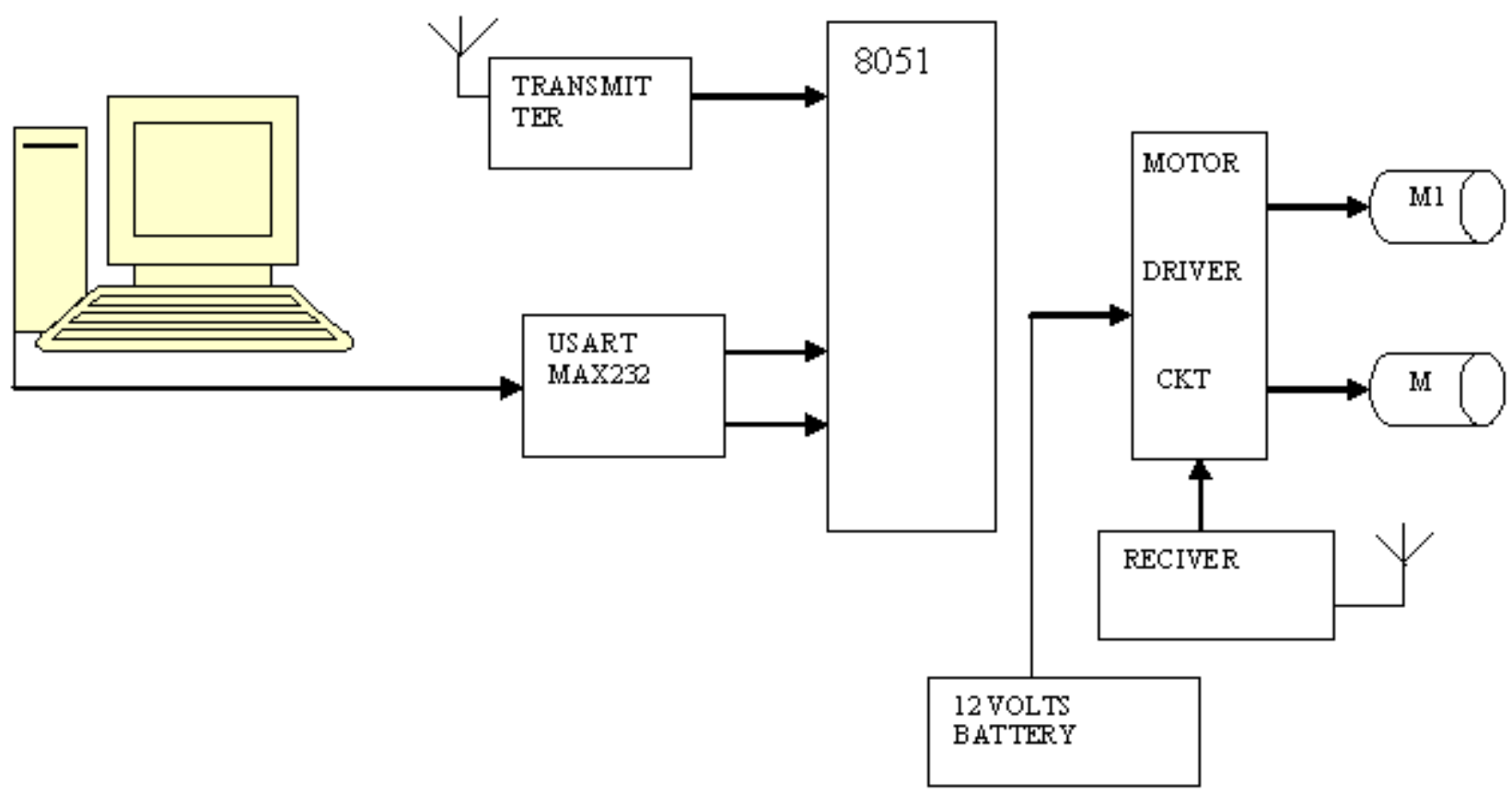

Fig. 1 Blog diagram

Block diagram contains the total overview of the system. In this, the computer is connected with microcontroller through RS232 via comport of CPU. Microcontroller receives the hexadecimal input from the computer and converts it into corresponding digital data for the purpose of transmitting, then the receiver will receive the 4-bit data and it is processed through H-bridge to control the motors.

\section{WORKING}

Since we are concerned with the computer part, here we discuss the working principal of computer program. We are using Microsoft Visual Studio to develop the front ends and also the coding using windows forms and $\mathrm{C \#}$ language as backend.

We are using grids to draw the path. Grid is mapped with proper scaling so that we can get the proper coordinates of path, so we are using 400X400 grid and numbers are scaled at the $\mathrm{X}$ and $\mathrm{Y}$ axis lines. Grid mapping and scaling is shown in figure 2 .

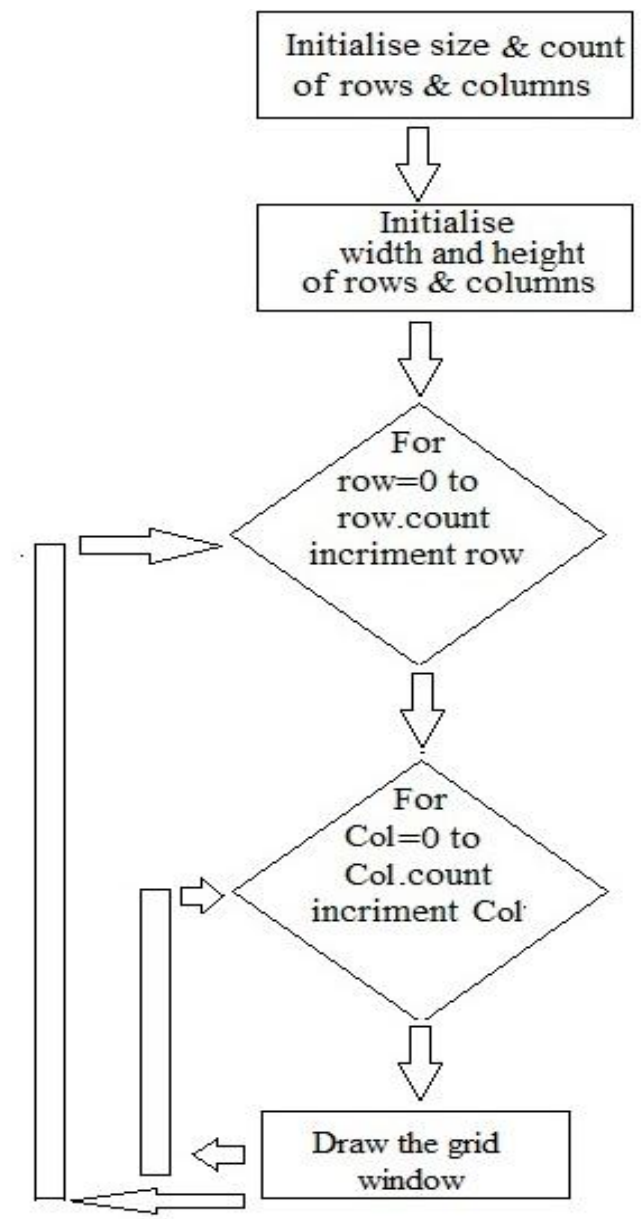

Fig. 2 Algorithm for window grid 
The above algorithm shows the image drawing window. Here we are considering the whole window as the small boxes of same size, which are arranged in the horizontal and vertical direction that is two directional way as like the pixel arrangement of any screen. Also mouse event handlers are implemented for writing the line on the grid i.e. mouse down, mouse up. As the mouse down, the pixel filling starts. Once the mouse is up the writing of pixel get stopped.

For the filling of next pixels we have used thread, the thread sleeps for the period of some microseconds so that it can select next pixel in that time span. Here we have applied an approximation algorithm. Function of approximation algorithm is to eliminate all the pixels which are repeated at same point. As the line drawing is completed, tracing mechanism starts.

\section{LINE TRACING ALGORITHM}

1. If current coordinate point is equal to previous coordinate point then it returns Start. . This means that there is no previous point obviously the robot will be in ideal state.
2. Current point is greater than previous point in $\mathrm{X}$ direction and current point is same as previous point in Y direction then it returns Right.

3. Current point is greater than previous point in $\mathrm{X}$ direction and current point is less than previous point in Y direction then it returns Forward Right.

4. Current point is equal to previous point in $\mathrm{X}$ direction and current point is less than previous point in $\mathrm{Y}$ direction then it returns Forward.

5. Current point is less than previous point in both $\mathrm{X}$ and Y direction then it returns Left.

6. Current point is less than previous point in $\mathrm{X}$ direction and current point is equal to previous point in $\mathrm{Y}$ direction then it returns Left.

7. Current point is less than previous point in $\mathrm{X}$ direction and current point is greater than previous point in $\mathrm{Y}$ direction then it returns Backward Right.

8. Current point is equal to previous point in $\mathrm{X}$ direction and current point is greater than previous point in $\mathrm{Y}$ direction then it returns backward.

9. Current point is greater than previous point in both $\mathrm{X}$ and $\mathrm{Y}$ direction then it returns Left.

10. Current coordinate is null then it returns Stop.

Graphical algorithm for line follower robot:
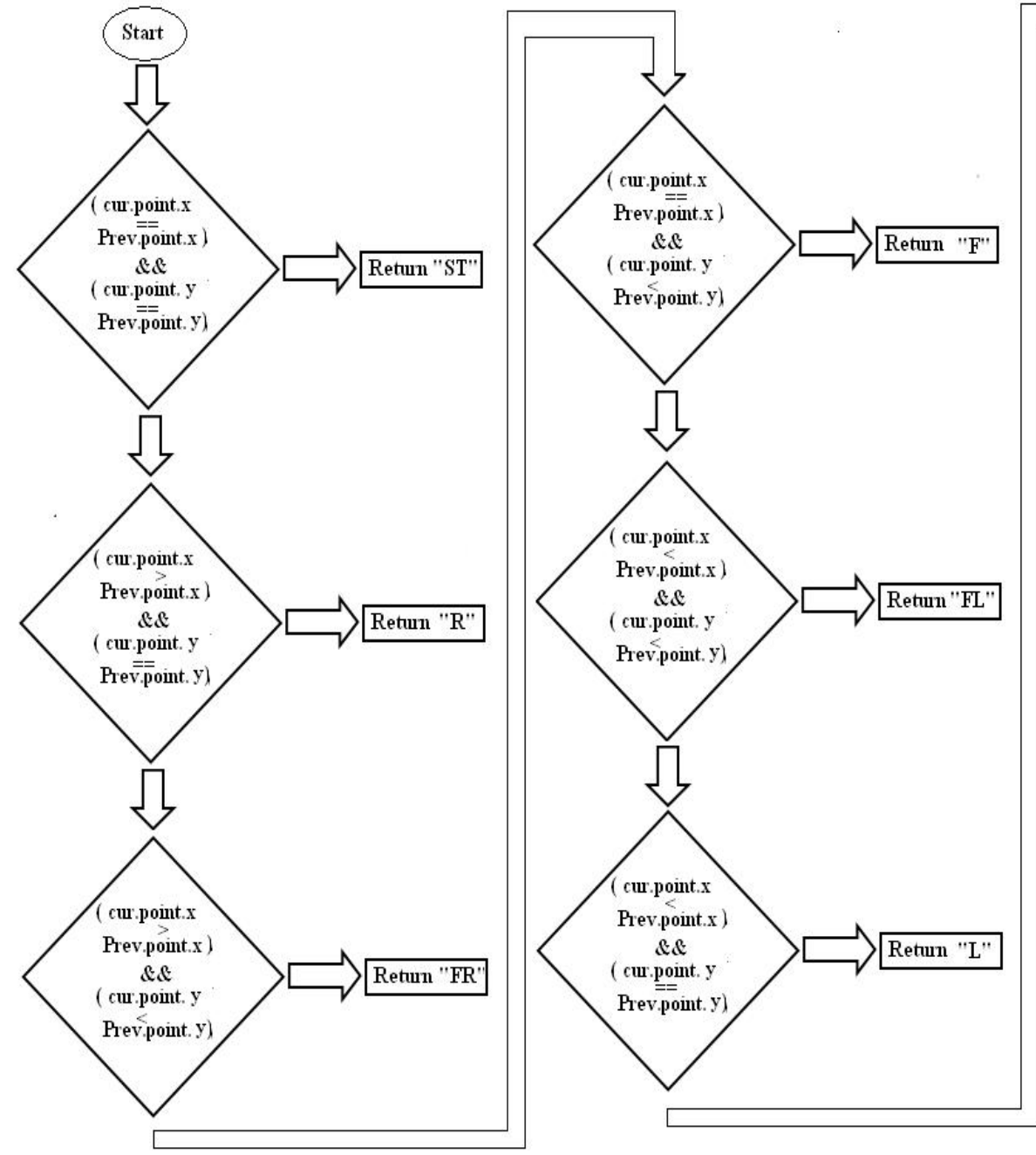

Fig 3.Line Tracing Algorithm

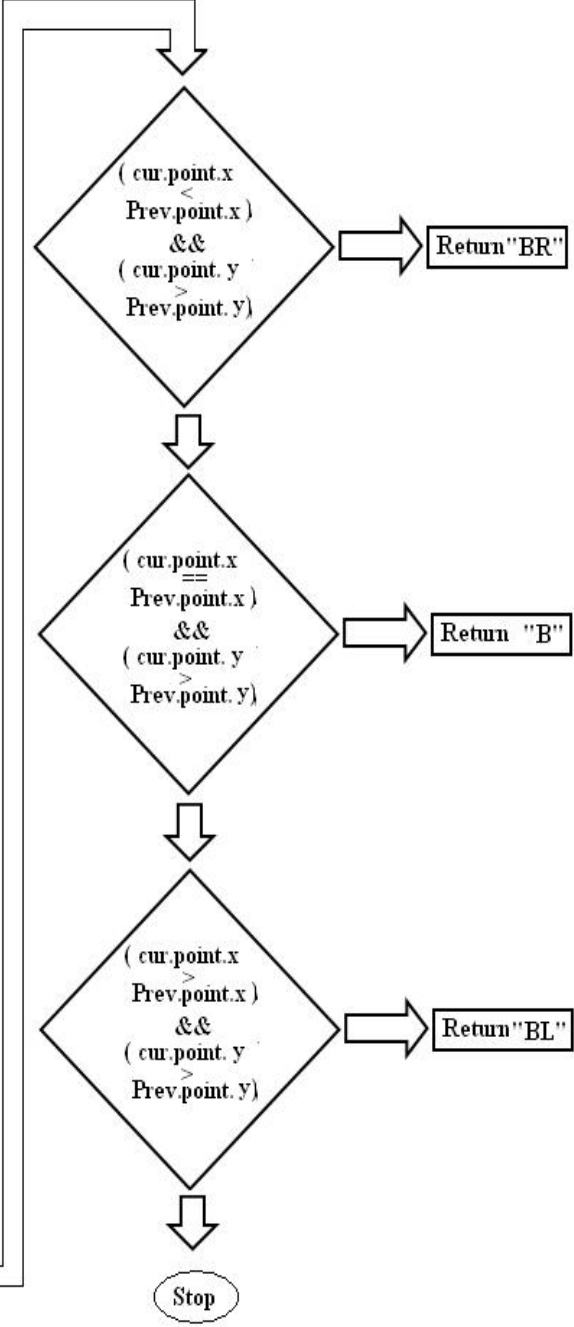

Stop 
Above figure shows the line tracer algorithm which is the main part of the project. This is the algorithm which is introducing first time in the field of computer controlled robot. This algorithm compares the current point with the previous point based on this the propulsion of the robot is determined.

\section{RESULT AND EXPERIMENT}

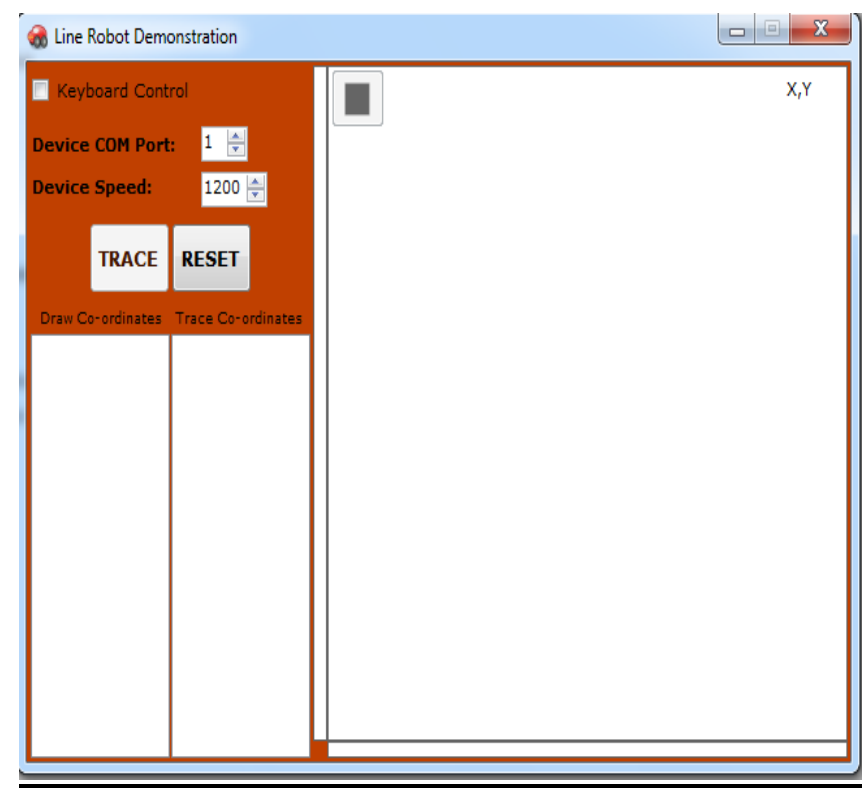

Fig 4.Initial view of executable file

The initial view of the GUI is shown in figure 4. The line drawing is done on the right path of window and two buttons are used for tracing and resetting the window for new line. Below shows the snap shot of window where we have drawn the line which is almost near to the rectangle. At the left side just below the trace button we can see that some numbers which represent the $\mathrm{X}$ and $\mathrm{Y}$ coordinates of the window.

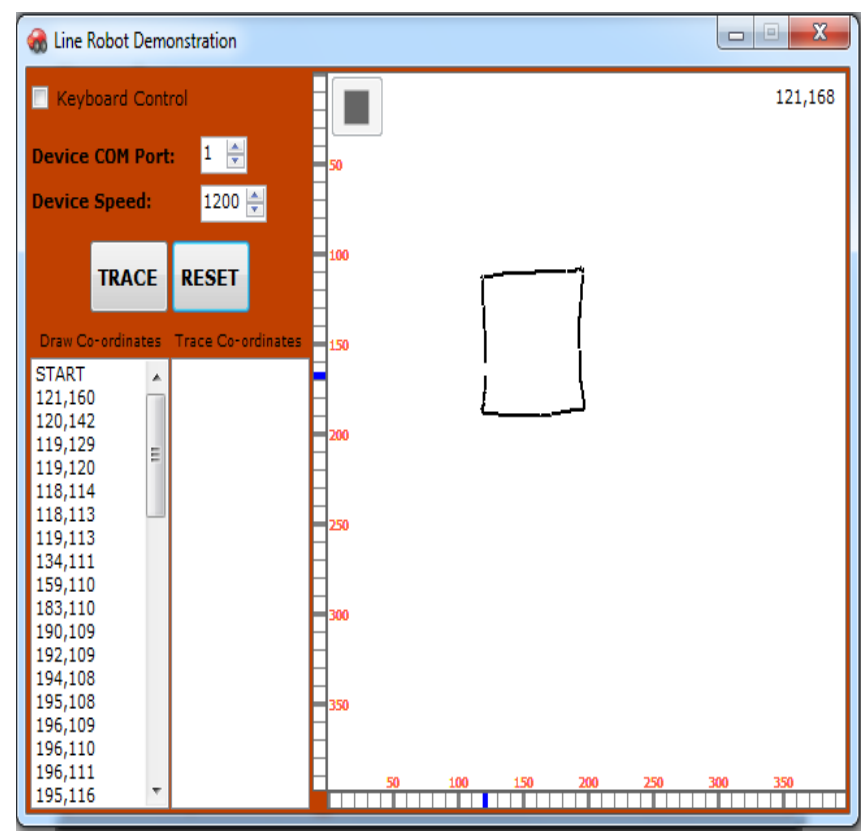

Fig.5.Before tracing rectangle drawing

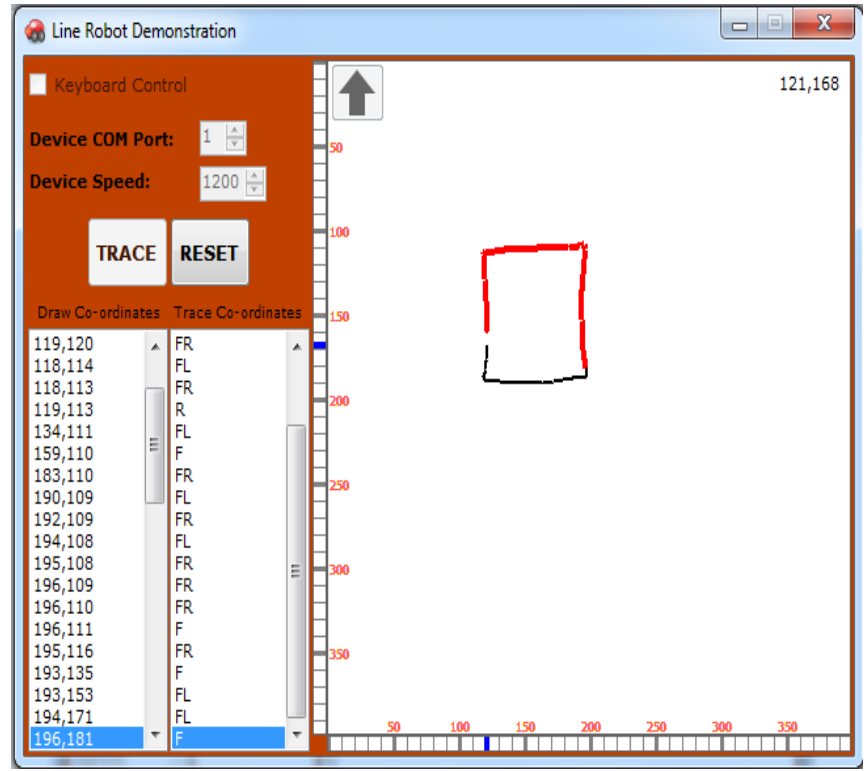

Fig.6 In-between tracing of rectangle

In figure 6 just below the reset button there is a list which contains the different alphabets which represents the different movements of robot as explained in the line tracer algorithm.

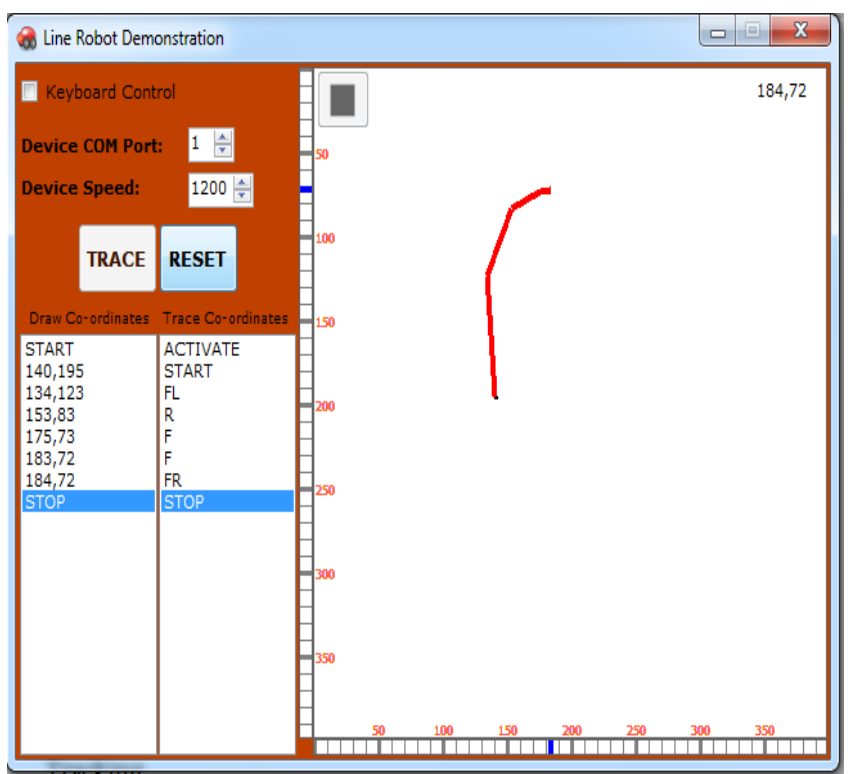

Fig 7 Complete operation from start to stop

In the figure 7 a curve is drawn which shows the complete operation of the robot from start to stop.

\section{CONCLUSION}

The aim of the project is to replace the sensor array with the computer and mobile technology i.e. the RF module for mobile navigation along with the computer to decide the direction. This has been successfully achieved. This is the advanced version of line follower robot or automated guided vehicle, more over we have implemented many new technologies like we have introduced the transmitters at the computer end along with the microcontroller at the same side. 


\section{FUTURE WORK}

Since this is the start of a new technology many changes have to make to get advancements in the system. Like during the drawing difficulties are obvious. This is the main disadvantage here.

The next thing to be implementing in this robot is elimination of the problem of computer program as a new project. As future work, we are thinking regarding the replacing of transmitters and receivers by transceivers at both the end. Till now it is one way communication, we are planning for two way communications, so as to inform the obstacles by the Robot to the computer and many more.

\section{APPLICATIONS}

\section{In Industries Automated Equipment Carriers}

In manufacturing companies or industries, there need to carry big loads or heavy materials from one location to another. This paper provides concepts to build such robots to carry these heavy materials and can be controlled by computer system.

\section{Building Entertaining Toys and Small Household}

\section{Applications}

Different toys can be built for the kids having wireless control system like remote and small household applications like Robotic vacuum cleaners and floor-washing robots that clean floors with sweeping cloth disposable cleaning for sweeping floor.

\section{Automated Cars}

Cars which run without driver can be built. These cars are capable of sensing its environment and navigating without human input.

\section{In Museums, used as Tour Guides and Other}

\section{Applications}

Some museums use telepresence robots to give a virtual guided tour of museums to encourage over world. These concepts can also be used to build such robots to help physical and virtual visitors to overcome language barriers by speaking in a language that they understand. Because of these advantages, most of the museums are interested to begin the use of robots for giving virtual tours to those who cannot travel due to physical disabilities.

\section{INNOVATIONS}

We have introduced the robot which is programmed and controlled at the computer end using microcontrollers. We have taken advantages of radio communication to mobile robot from the base station i.e. the computer to guide the path.

\section{REFERENCES}

[1] M. P. Groover, "Automation, Production System and Computer Integrated Manufacturing”, New York: Prentice Hall, 1987.

[2] M. Kaighobadi, and K. Venkatesh, "Flexible Manufacturing Systems: an overview", International Journal of Operations \& Production Management, vol. 14, pp.26-49, 1994.

[3] G. G. Kost, and R. Zdanowicz, "Modeling of manufacturing systems and robot motions", Journal of Materials Processing Technology, vol. 164-165, pp. 1369-1378, 2005.

[4] S. Takukawa, "The use of Simulation in ActivityBased Costing For Flexible Manufacturing Systems", Proc of the 1997 Winter Simulation Conference, pp. 793-800, 1997.

[5] P. E. Guy, and A. Castleberry, The AGV Handbook. Ann Arbor, Michigan: Braun-Brumfield, Inc., 1991.

[6] Wikipedia.

[7] Chapter 2 Fundamentals of Robotics, This sample chapter is for review purposes. Copyright (C) The Goodheart-Willcox Co.

[8] Command Based Line Following Robot using RF Technology -AnjumKhaliqueBhatti, Muhammad IqbalBhatti, Kamran Khowaja and Asadullah Shah Department of computer science ,Malaysia. Journal of Advanced Computer Science and Technology Research 1 (2011) 25-35.

[9] Design of Automatic Steering Control and Adaptive Cruise Control of Smart Car, D.Sivaraj,K.R.Radhakrishnan, A.Kandaswamy, J.Prithiviraj,S.Dinesh, Babu,T.J.Krishanth, International Conference on VLSI, Communication \& Instrumentation (ICVCI) 2011 Proceedings published by International Journal of Computer Applications ${ }^{\circledR}$ (IJCA).

[10] Design and implementation ofdouble line follower robot, v hymavathi, g vijay kumar, V Hymavathi et al. / International Journal of Engineering Science and Technology (IJEST). 